\title{
Marker-less Respiratory Motion Modeling Using the Microsoft Kinect for Windows
}

\author{
F. Tahavori ${ }^{a}$, M. Alnowami ${ }^{b}$ and K. Wells ${ }^{a}$ \\ ${ }^{a}$ Centre for Vision, Speech and Signal Processing, University of Surrey, Guildford, UK, \\ GU2 7XH; \\ ${ }^{b}$ Nuclear Engineering Department, King Abdulaziz University, P.O. Box 80204 Jeddah, \\ Kingdom of Saudi Arabia.
}

\begin{abstract}
Patient respiratory motion is a major problem during external beam radiotherapy of the thoracic and abdominal regions due to the associated organ and target motion. In addition, such motion introduces uncertainty in both radiotherapy planning and delivery and may potentially vary between the planning and delivery sessions. The aim of this work is to examine subject-specific external respiratory motion and its associated drift from an assumed average cycle which is the basis for many respiratory motion compensated applications including radiotherapy treatment planning and delivery. External respiratory motion data were acquired from a group of 20 volunteers using a marker-less 3D depth camera, Kinect for Windows. The anterior surface encompassing thoracic and abdominal regions were subject to principal component analysis (PCA) to investigate dominant variations. The first principal component typically describes more than $70 \%$ of the motion data variance in the thoracic and abdominal surfaces. Across all of the subjects used in this study, $58 \%$ of subjects demonstrate largely abdominal breathing and 33\% exhibited largely thoracic dominated breathing. In most cases there is observable drift in respiratory motion during the 300s capture period, which is visually demonstrated using Kernel Density Estimation. This study demonstrates that for this cohort of apparently healthy volunteers, there is significant respiratory motion drift in most cases, in terms of amplitude and relative displacement between the thoracic and abdominal respiratory components. This has implications for the development of effective motion compensation methodology.
\end{abstract}

\section{INTRODUCTION}

Patient respiratory motion is a major problem during external beam radiotherapy of the thoracic and abdominal regions due to the associated organ and target motion. In addition, such motion introduces uncertainty in both radiotherapy planning and delivery ${ }^{1}$ and may potentially vary between the planning and delivery sessions. ${ }^{2}$ Such associated error in the defined treatment margin may lead to unwanted irradiation of healthy tissue and compromised dose delivery to the target region. Motion management is thus crucial in diagnostic imaging and therapy. Keall et al. ${ }^{3}$ proposed using $4 \mathrm{D}$ radiotherapy which considers the temporal dimension in both the planning acquisition and treatment to address these difficulties. ${ }^{3,4}$ In $4 \mathrm{D}$ RT, respiratory motion modeling is now gaining significant interest although tracking is most often undertaken using a set of fiducial markers. ${ }^{4}$ Some researchers have used a CT volume obtained in cine mode ${ }^{5}$ to extract the breathing signal during respiratory cycle but this method has the drawback of assuming an averaged invariant respratory pattern. Others have used $4 \mathrm{D} \mathrm{CT}$ or $4 \mathrm{D}$ MRI to extract respiratory motion. ${ }^{4,6}$ However application of these methods is limited to extra dose in CT imaging and slow acquisition during MRI. Wireless tracking offers a complimentary approach to provide actual internal target motion as used in the Calypso system. ${ }^{4}$ Nevertheless, an alternative approach that we propose, that is both marker-less and non-ionising, involves using a 3D depth camera, such as the Microsoft Kinect for Windows to capture and analyse external respiratory motion.

The intrinsic respiratory signal has several basic parameters such as amplitude, frequency and phase. These may vary over time and may exhibit inter- and intra patient variation. This latest study focuses on subject

Further author information: (Send correspondence to F.T.)

F.T.: E-mail: f.tahavori@surrey.ac.uk, Telephone: +44 (0)1483 689856

K.W.: E-mail: k.wells@surrey.ac.uk, Telephone: +44 (0)1483686036 
specific external respiratory motion analysis of such inter- and intra subject parameters. Moreover, we examine respiratory motion drift during normal breathing. In doing so, we have imaged a group of volunteers using a marker-less 3D depth camera, Kinect for Windows and undertaken a ROI-based Principal Component Analysis $(\mathrm{PCA})$ to investigate the dominant variation.

\section{METHODOLOGY AND RESULTS}

\subsection{Volunteer Preparation}

For this study, a group of twenty healthy volunteers participated, 6 females and 14 males. For each volunteer, respiratory motion was captured individually using a Kinect for Windows camera system on 3 separate sessions, each separated by 1 week. The Kinect can capture the 3D motion of the anterior surface information from a continuously projected infrared light pattern, providing a depth map that can change with motion in the scene. Previous work at our institution has shown that the Kinect for Windows error can be less than $1 \mathrm{~mm}$ at $80 \mathrm{~cm}$ from the camera. ${ }^{7}$ The Kinect unit was placed above the volunteer's chest in the supine position where the distance between the chest surface and camera was approximately $80 \mathrm{~cm}$. Respiratory motion data were then captured for $300 \mathrm{~s}$. No instruction was provided regarding breathing style and normal tidal breathing was recorded for all volunteers at 10 frames per second. Figure 1(a) illustrates the experimental arrangement.

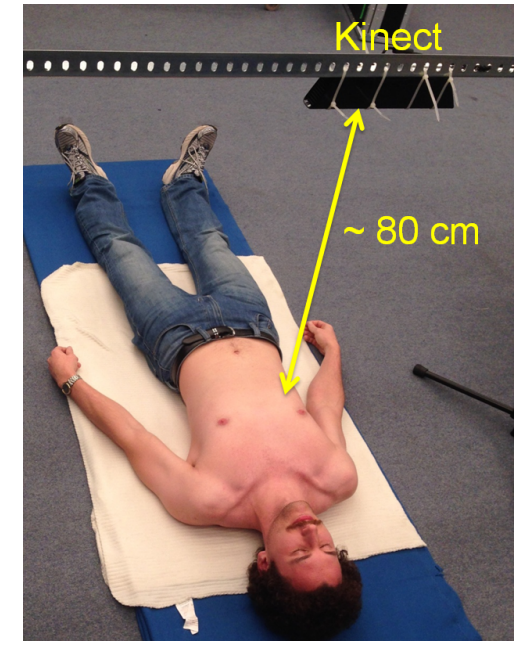

(a)

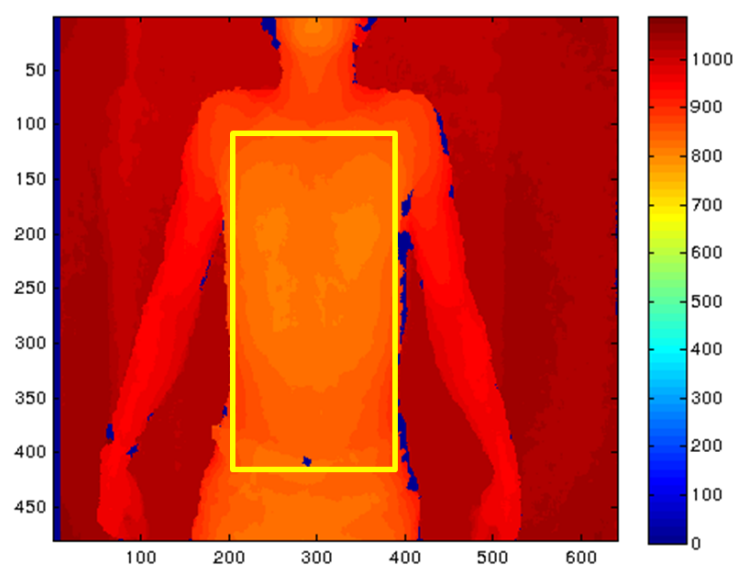

(b)

Figure 1. (a) An example of a volunteer in supine position where external respiratory motion was captured using a Kinect for Windows operating in near-mode. (b) Exemplar of depth map produced by the Kinect with a ROI selected encompassing the thoracic and abdominal surfaces.

\subsection{Data}

Respiratory motion was captured and analysed using in-house software developed in the Matlab environment. The amplitude, gradient and phase of respiratory motion were extracted from each data set. Figure 2 (A and D) illustrates two images representing the standard deviation of the displacement seen at each pixel over the $300 \mathrm{~s}$ acquisition across two of the subjects (upper subject with one of the most consistent results, lower subject with one of the highest level of temporal variation). This demonstrates respiratory variance dominated by abdominal motion in Figure 2(A), whereas in Figure 2(D) similar levels of variation are seen in the central abdominal/thoracic regions, but with greater lateral thoracic variance.

\subsection{Preprocessing of Captured Data (Data Characterization) and Preliminary Results}

\subsubsection{Single ROI PCA}

A Region Of Interest (ROI) was selected on the surface of each subject encompassing the whole thoracic and abdominal surfaces (see Figure 2, A and D). Figure 3 represents the histogram of displacement from the mean over 300 s for the ROIs shown in Figure 2 for 2 volunteers in 3 different weeks. 

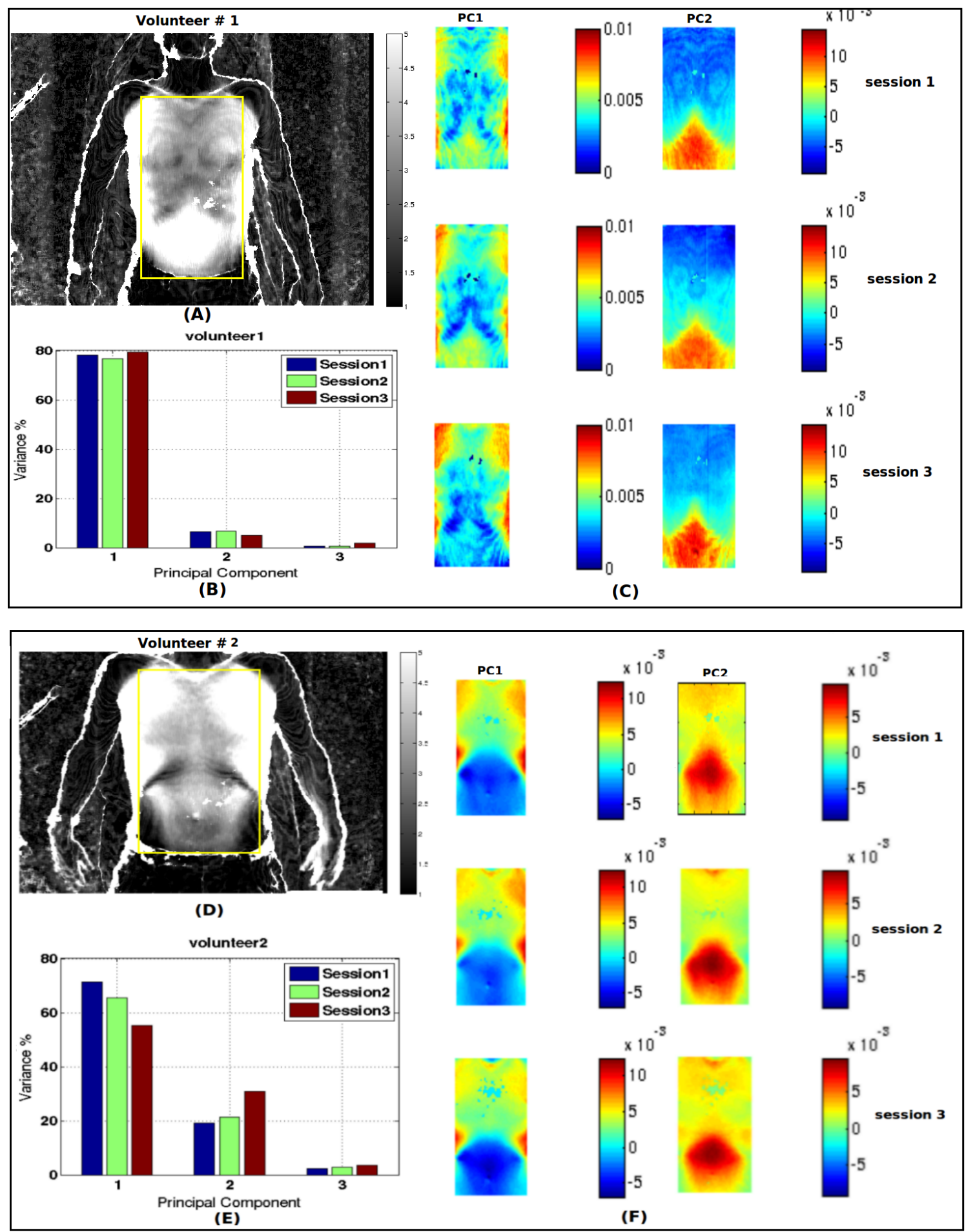

Figure 2. Two examples of the pixel wise standard deviation image of volunteer's anterior surface as extracted from the Kinect depth map (image A, D) for the volunteer (volunteer \#1 shown in Table 1) with one of the most consistent result (A) and for the volunteer (volunteer \#2 shown in Table 1) with one of the highest level of temporal variation (D), captured using a Kinect for Windows. The change in the eigenvalues over 3 sessions for these 2 individuals is shown in (B) and (E). $(\mathrm{C})$ and $(\mathrm{F})$ show the first two eigenimages for those two volunteers demonstrating change in the abdominal and thoracic components. 
PCA was undertaken on the temporal data defined by the aforementioned single ROI to illustrate inter-session change in the external respiratory motion. The ROI on the anterior surface was defined to study the external surface motion parameters in more detail. This group motion can be described by Equations 1 for the whole surface $\left(W_{s}\right)$ :

$$
W_{s}=\left[F_{1}, F_{2}, F_{3}, \ldots, F_{\text {tmax }}\right] \text {, }
$$

where $F_{t}$ is a column matrix at time $t$ and includes the depth value of each pixel at each time sample, $t$ in the selected abdominal-thoracic ROI. PCA was applied to $W_{s}$. Figure 2 (B and E) demonstrates that the first principal component describes more than $75 \%$ of data variance in the thoracic and abdominal surfaces for the consistent subject seen in Figure 2(A), and around $55 \%$ of data variance in the thoracic and abdominal surfaces for the more inconsistent subject seen in Figure 2(D) across all three sessions. Table 1 summaries the PCA analysis undertaken for the complete volunteer cohort.

Figure $2(\mathrm{C}$ and $\mathrm{F}$ ) shows the first two principal components images, while each rows represents first, second and third sessions PCA results. The first principal component in Figure 2 (C) illustrates the ensemble motion of the entire chest surface and the second principal component showing the relative variation of the abdominal/thoracic motion component.

\subsubsection{Multi-ROI PCA}

In order to better illustrate the relative motion of the abdominal and thoracic components, a seperate multi-ROI analysis was undertaken. The choice of ROIs is similar to the approach taken in multi-marker based analysis ${ }^{8}$ but here each ROI acts as a virtual marker to separate these two motion components (abdominal and thoracic). 16 Region Of Interests (ROIs) were selected on the thoracic and abdominal surfaces for each subject (see Figure 4). The mean pixel displacement value within each ROI of approximate $16 x 16$ pixels was used at each time point for the analysis. The 8 upper most ROIs were then selected to produce a representative number for the thoracic displacement component, $T_{s}$. This was repeated for the lower 8 ROIs to produce a representative number for the abdominal displacement component, $A_{s}$. Figure 5 represents the mean displacement over $300 \mathrm{~s}$ for four ROIs specified by blue circles in Figure 4. As can be seen in Figure 5, $T_{s}$ and $A_{s}$ are in-phase for the subject seen in Figure 2(A) and out-phase for the subject seen in Figure 2(D).

This group motion can be described by Equations 2 and 3 for thoracic $\left(T_{s}\right)$ and abdominal surface $\left(A_{s}\right)$ respectively:

$$
\begin{aligned}
& T_{s}=\left[F_{1}, F_{2}, F_{3}, \ldots, F_{\text {tmax }}\right], \\
& A_{s}=\left[F_{1}, F_{2}, F_{3}, \ldots, F_{t \max }\right],
\end{aligned}
$$

where $F_{t}$ is a column matrix at time $t$ and includes the mean depth value at each time sample, $t$, of 8 ROIs for $T_{s}$ and 8 ROIs for $A_{s}$. PCA was applied to each of $T_{s}$ and $A_{s}$. Figure 6 demonstrates that when these two components are separated, then the first principal component describes more than $98 \%$ of data variance in the thoracic and abdominal surfaces for the subject seen in Figure 2(A), and more than $98 \%$ and $90 \%$ of data variance in the thoracic and abdominal surfaces for the subject seen in Figure 2, D).

The original data were then reconstructed from the projection of the data onto the first principal component using a least squares approximation. ${ }^{9}$ Using the first eigenvector, the temporal displacement of the reconstructed thoracic surface was plotted against that of the reconstructed abdominal surface. This can be used to study the relative displacement between thoracic and abdominal motion and thereby any drift in this behavior (see Figure 7). The colour coding demonstrates the temporal evolution of the signal from volunteer \#1, from blue (start) to red (end). Figure 7 (left) demonstrates that even during very uniform breathing, there is significant temporal drift in the amplitude and relative displacement between the thoracic and abdominal respiratory components. For the volunteer number 2 in Table 1 (seen in Figure 2, D), clearly very different patterns of breathing are seen at the beginning of acquisition (blue) compared to the end. 


\subsection{Gaussian Kernel Density Estimation}

Gaussian Kernel Density Estimation has been used as a convenient method of visualising the motion data of the abdominal and thoracic components produced from PCA using $T_{s}$ (Equation 2) and $A_{s}$ (Equation 3), and characterising these in terms the model proposed by Alnowami et al. ${ }^{8}$ Figure 8 shows exemplar KDE histograms produced from volunteers 1 and 8 , each separated by 1 week.

\section{CONCLUSION AND FUTURE WORK}

External surface respiratory motion was captured using Kinect for Windows and PCA was applied to an ROI encompassing the complete anterior surface. The results demonstrate various styles of breathing using 2 eigenvectors for thoracic and the abdominal surfaces. Of the 20 subjects studied we observed a few subjects where the first and second eigenimages were out-phase. One subject presented with the most consistent breathing style where the first and second eigenvectors were in the same direction. Across all of the subjects used in this study, $58 \%$ demonstrate largely abdominal breathing and 33\% exhibit thoracic dominated breathing. In each case results of temporal respiratory drift, and changes during and between different capture sessions were quantified using Kernel Density Estimation methods.

\section{REFERENCES}

[1] Ozhasoglu, C. and Murphy, M. J., "Issues in respiratory motion compensation during external-beam radiotherapy," International Journal of Radiation Oncology*Biology*Physics 52(5), 1389-1399 (2002).

[2] McClelland, J. R., Hughes, S., Modat, M., Qureshi, A., Ahmad, S., Landau, D. B., Ourselin, S., and Hawkes, D. J., "Inter-fraction variations in respiratory motion models," Physics in Medicine and Biology 56(1), 251 (2011).

[3] Keall, P. J., Mageras, G. S., Balter, J. M., Emery, R. S., Forster, K. M., Jiang, S. B., Kapatoes, J. M., Low, D. A., Murphy, M. J., Murray, B. R., Ramsey, C. R., Herk, M. B. V., Vedam, S. S., Wong, J. W., and Yorke, E., "The management of respiratory motion in radiation oncology report of AAPM task group 76," Medical Physics 33(10), 3874-3900 (2006).

[4] Fayad, H., Pan, T., Pradier, O., and Visvikis, D., "Patient specific respiratory motion modeling using a 3D patient's external surface," Medical Physics 39(6), 3386-3395 (2012).

[5] McClelland, J. R., Blackall, J. M., Tarte, S., Chandler, A. C., Hughes, S., Ahmad, S., Landau, D. B., and Hawkes, D. J., "A continuous 4D motion model from multiple respiratory cycles for use in lung radiotherapy," Medical Physics 33(9), 3348-3358 (2006).

[6] Ledesma-Carbayo, M., Bajo, A., Marta, C., Perez-David, E., Garcia-Fernandez, M., Desco, M., and Santos, A., "Fully automatic cardiac motion estimation from tagged MRI using non-rigid registration techniques," in [Computers in Cardiology, 2006], 305-308 (2006).

[7] Tahavori, F., Elangovan, P., Alnowami, M., Yamani, A., Donovan, E., and Wells, K., "Assessment of Microsoft Kinect Technology for patient monitoring during External Beam Radiotherapy," in [Nuclear Science Symposium Conference Record (NSS/MIC), 2013 IEEE], (October 2013).

[8] Alnowami, M., Lewis, E., Wells, K., and Guy, M., "Inter- and intra-subject variation of abdominal vs. thoracic respiratory motion using Kernel Density Estimation," in [Nuclear Science Symposium Conference Record (NSS/MIC), 2010 IEEE], 2921-2924 (2010).

[9] Zhang, Y., Yang, J., Zhang, L., Court, L. E., Balter, P. A., and Dong, L., "Modeling respiratory motion for reducing motion artifacts in 4D CT images," Medical Physics 40(4), 041716 (2013). 
Table 1. PCA analysis undertaken for the entire volunteer cohort, session 1 is a colour coded pink, session 2 color coded green, session 3 color coded in blue. The first principal component captures the ensemble motion of the entire chest surface, while the second principal component represents secondary motion, showing the variation of the dominant abdominal/thoracic motion component.

\begin{tabular}{|c|c|c|c|}
\hline Volunteer\# & Gender & $\begin{array}{l}\text { Single ROI me thod } \\
\text { FirstEig envalue }\end{array}$ & $\begin{array}{l}\text { Single Rol me thod } \\
\text { Second Eig envalue }\end{array}$ \\
\hline \multirow[t]{3}{*}{1} & \multirow[t]{3}{*}{$\mathrm{M}$} & 78.22 & 6.51 \\
\hline & & 81.66 & 7.24 \\
\hline & & 81.01 & 6.07 \\
\hline \multirow[t]{3}{*}{2} & \multirow[t]{3}{*}{$\mathrm{M}$} & 71.33 & 19.25 \\
\hline & & 67.16 & 23.89 \\
\hline & & 58.88 & 31.23 \\
\hline \multirow[t]{3}{*}{3} & \multirow[t]{3}{*}{$\mathrm{M}$} & 66.18 & 15.95 \\
\hline & & 53.21 & 17.13 \\
\hline & & 57.95 & 13.52 \\
\hline \multirow[t]{3}{*}{4} & \multirow[t]{3}{*}{$\mathrm{F}$} & 62.55 & 1.42 \\
\hline & & 49.38 & 10.78 \\
\hline & & 47.46 & 3.50 \\
\hline \multirow[t]{3}{*}{5} & \multirow[t]{3}{*}{$\mathrm{F}$} & 76.33 & 5.04 \\
\hline & & 73.59 & 7.56 \\
\hline & & 67.69 & 3.93 \\
\hline \multirow[t]{3}{*}{6} & \multirow[t]{3}{*}{$\mathrm{M}$} & 93.19 & 1.61 \\
\hline & & 64.03 & 26.15 \\
\hline & & 93.22 & 1.02 \\
\hline \multirow[t]{3}{*}{7} & \multirow[t]{3}{*}{$\mathrm{F}$} & 83.92 & 4.61 \\
\hline & & 81.01 & 5.52 \\
\hline & & 78.69 & 3.13 \\
\hline \multirow[t]{3}{*}{8} & \multirow[t]{3}{*}{$\mathrm{F}$} & 40.59 & 11.35 \\
\hline & & 26.74 & 5.23 \\
\hline & & 29.64 & 5.40 \\
\hline \multirow[t]{3}{*}{9} & $\mathrm{M}$ & 62.89 & 2.47 \\
\hline & & 49.88 & 10.88 \\
\hline & & 60.69 & 2.33 \\
\hline 10 & $\mathrm{~F}$ & 50.39 & 4.50 \\
\hline & & 56.37 & 2.30 \\
\hline & & 60.00 & 1.20 \\
\hline 11 & $\mathrm{M}$ & 83.22 & 0.68 \\
\hline & & 83.55 & 1.01 \\
\hline & & 72.67 & 2.93 \\
\hline 12 & $\mathrm{~F}$ & 53.72 & 9.67 \\
\hline & & 53.33 & 9.81 \\
\hline & & 49.00 & 16.38 \\
\hline 13 & $M$ & 62.64 & 6.94 \\
\hline & & 71.94 & 5.90 \\
\hline & & 73.82 & 6.13 \\
\hline 14 & $M$ & 86.47 & 4.27 \\
\hline & & 89.74 & 5.09 \\
\hline & & 76.22 & 11.07 \\
\hline 15 & $M$ & 72.76 & 14.30 \\
\hline & & 68.29 & 6.99 \\
\hline & & 67.79 & 1.40 \\
\hline 16 & $M$ & 48.41 & 4.70 \\
\hline & & 54.72 & 3.70 \\
\hline & & 63.14 & 3.10 \\
\hline 17 & $M$ & 90.04 & 1.55 \\
\hline & & 90.67 & 1.19 \\
\hline & & 87.10 & 0.76 \\
\hline 18 & $M$ & 81.15 & 0.81 \\
\hline & & 85.11 & 0.70 \\
\hline & & 83.70 & 0.60 \\
\hline 19 & $M$ & 87.80 & 6.80 \\
\hline & & 94.07 & 1.10 \\
\hline & & 90.93 & 1.36 \\
\hline 20 & $M$ & 55.74 & 7.30 \\
\hline & & 51.43 & 10.68 \\
\hline & & 52.90 & 14.33 \\
\hline
\end{tabular}



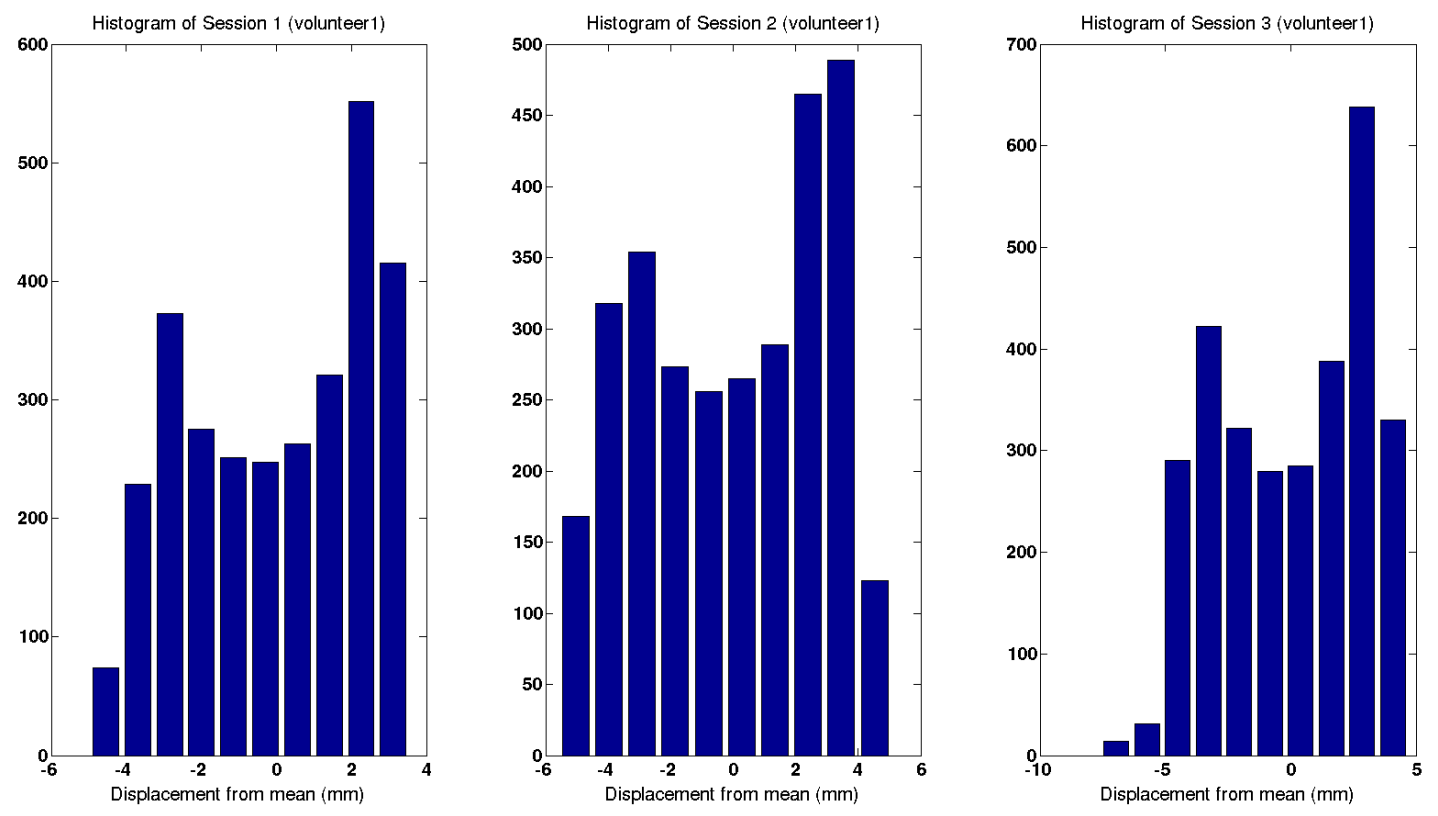

(a)
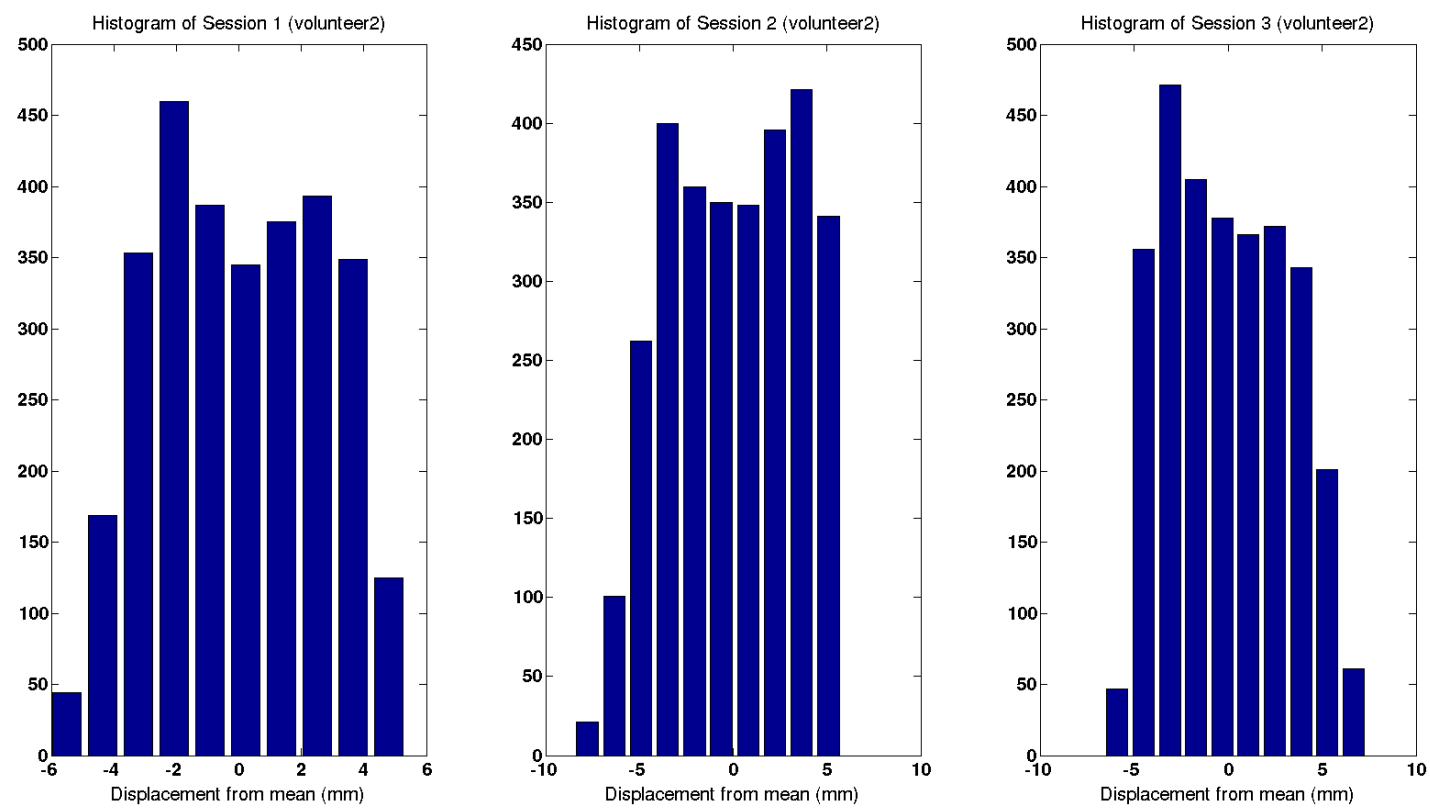

(b)

Figure 3. The histogram of displacement from the mean over $300 \mathrm{~s}$ was obtained for a ROI shown in figure 2 for the subject 1 in (a) and subject 2 in (b). This shows good inter session histogram consistency in volunteer 1 and correspondingly poor inter-session consistency for volunteer 2 . 


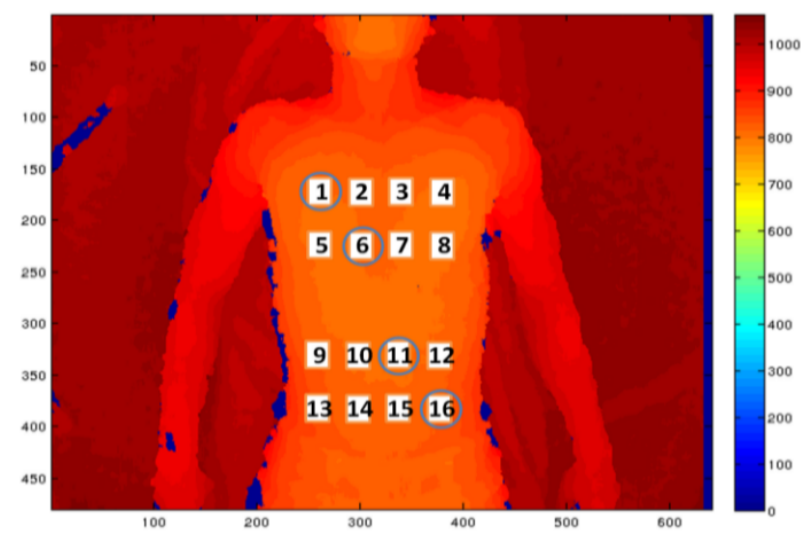

(a)

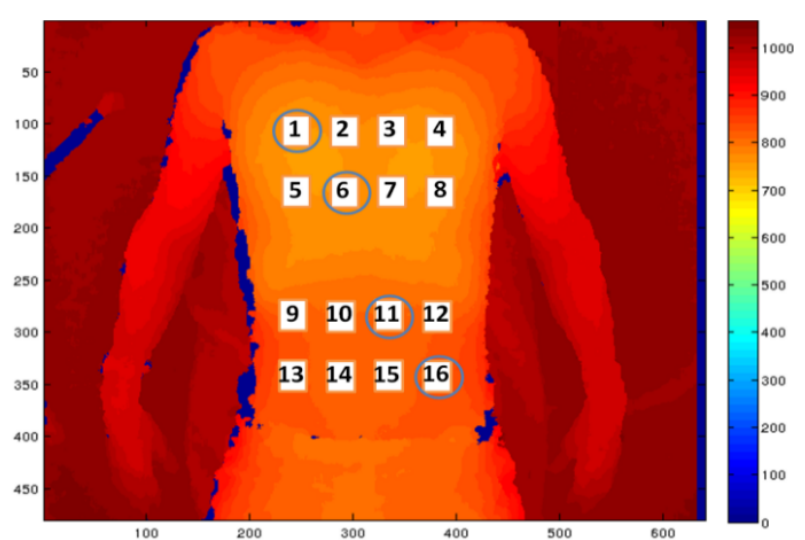

(b)

Figure 4. Exemplars of depth maps produced by the Kinect with ROIs selected on the thoracic and abdominal surfaces, and numbered as shown above, (a) for the volunteer with most consistent result and (b) for the volunteer with highest level of temporal variation.
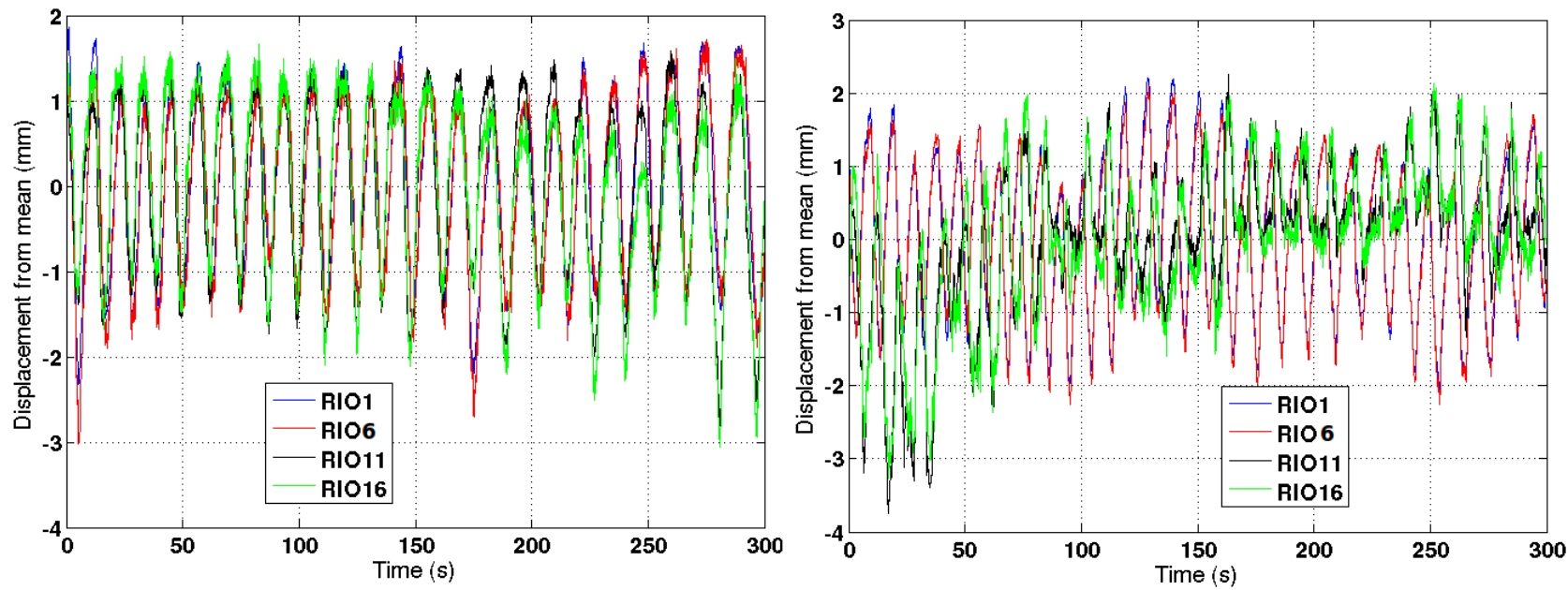

Figure 5. The displacement from the mean was plotted over $300 \mathrm{~s}$ for four ROIs shown in figure 4 for the subject seen in Figure 4(a) on left and the subject seen in Figure 4(b) on right. This shows the relative difference in displacement in the thoracic (ROI 1,6) and abdominal (ROI 11, 16) components. 

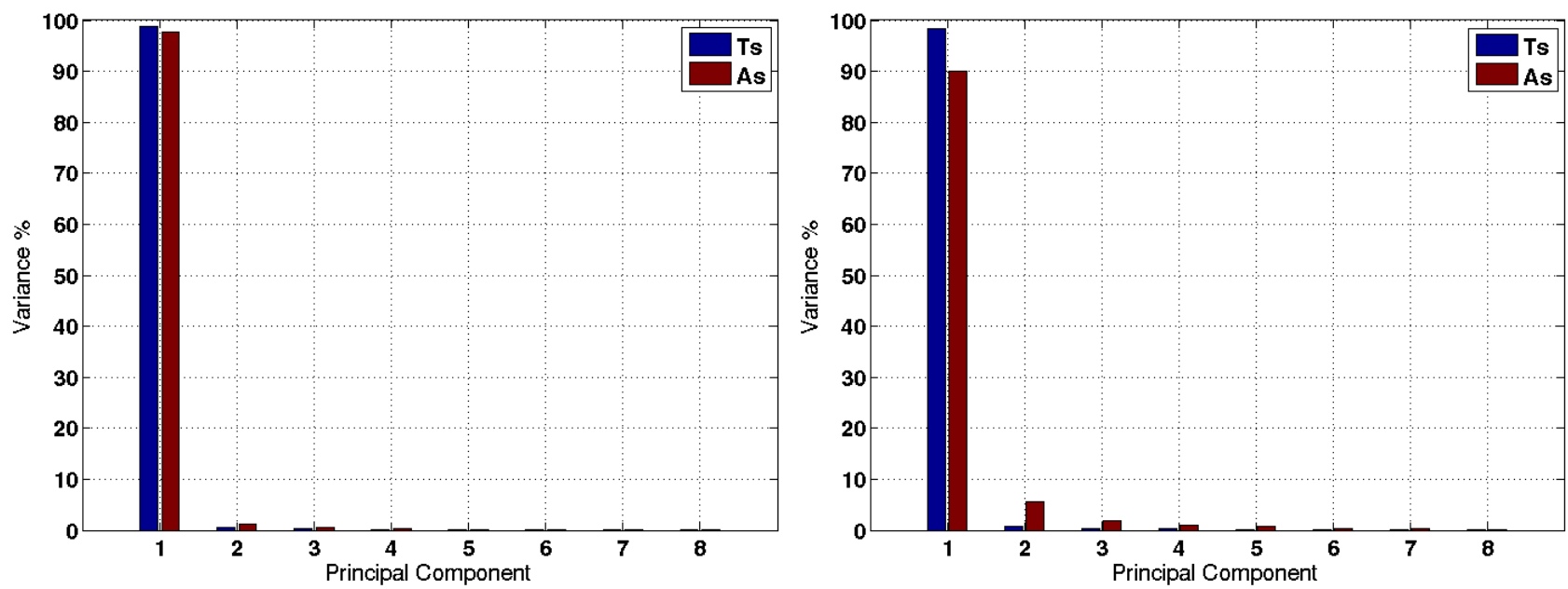

Figure 6. Eigenvalues produced from PCA of the $T_{s}$ and $A_{s}$ regions from two volunteers seen in Figure 4 . This shows that the virtual marker based ROI approach captures $\geq 90 \%$ of the motion in first principal components of the $T_{s}$ and $A_{s}$ motion.
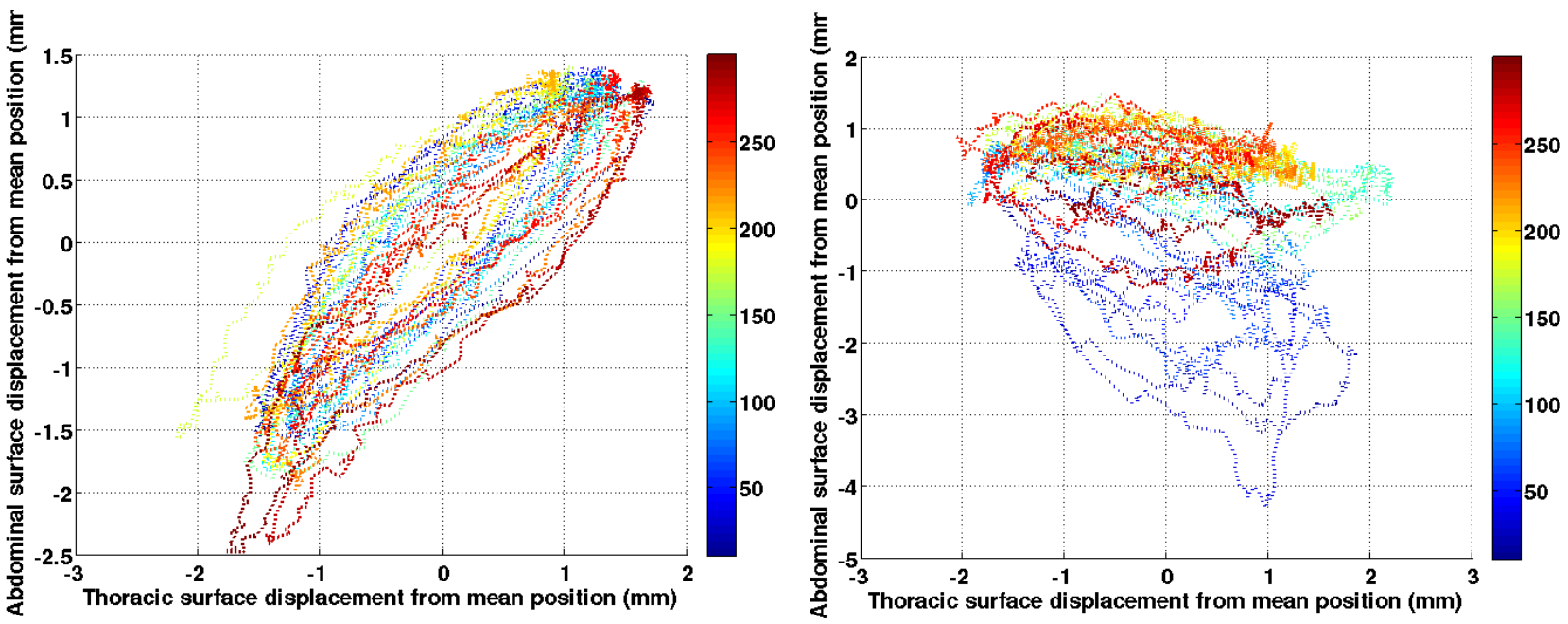

Figure 7. The axes describe the variation in the reconstructed of the original displacement in the subspace of thoracic and abdominal surfaces within $300 \mathrm{~s}$ with the subject seen in Figure 2 (A and D) respectively. 

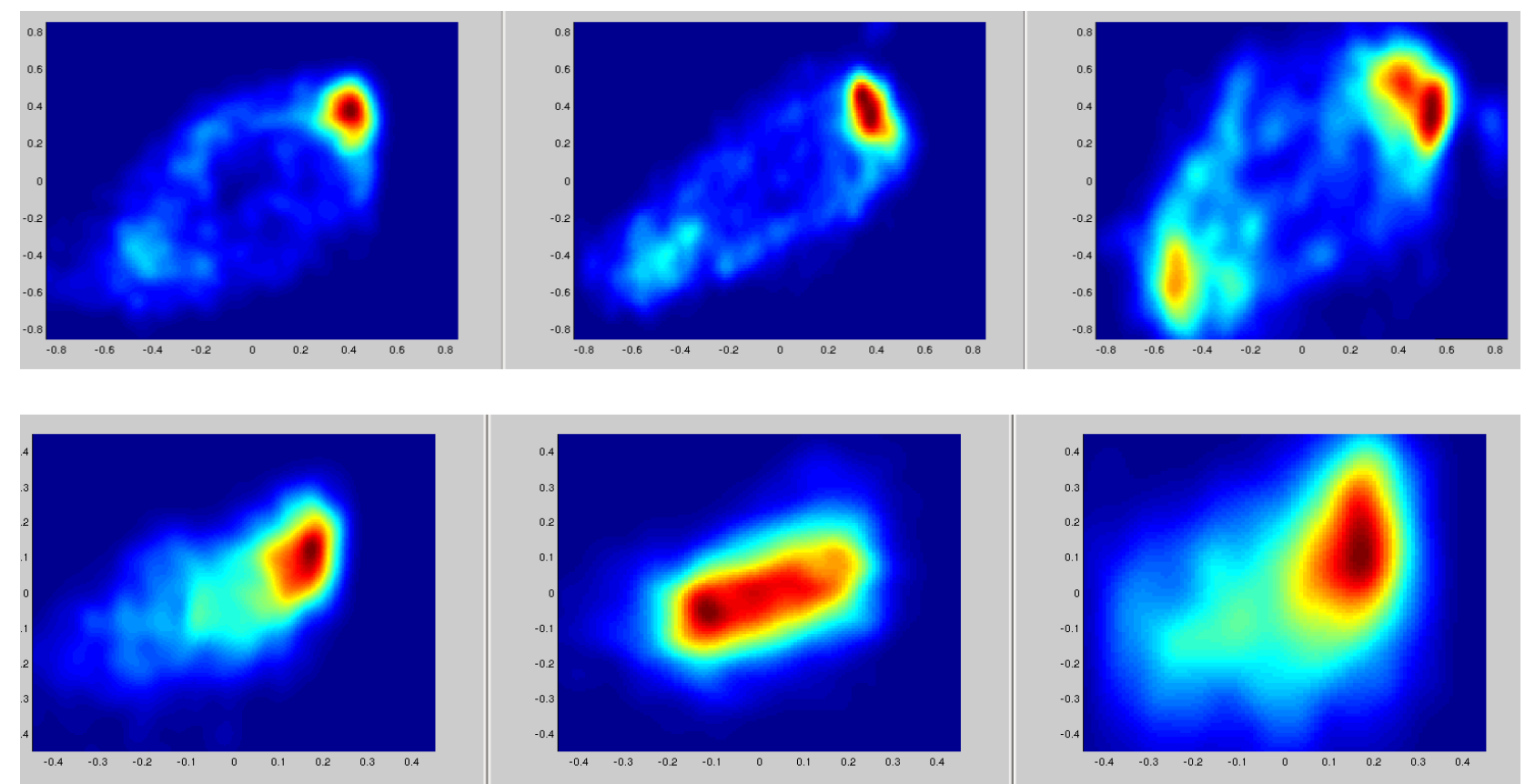

Figure 8. Plotting $T_{s}$ and $A_{s}$ on the x,y axes respectively, and then producing normalised KDE histograms graphically illustrate the change in respiratory motion pattern occurring in volunteers 1(top) and 8(bottom) volunteers across session 1(left), session 2(middle) and session 3(right) each separated by 1 week. 